\title{
On the Quantum Mechanics of Collision Processes.
}

\author{
[Preliminary communication. ${ }^{1}$ ]
}

By Max Born, Göttingen.

(Received June 25, 1926.)

An examination of collision processes indicates that Schrödinger's quantum mechanics describes not only stationary states, but also quantum jumps.

Heisenberg's quantum mechanics calculates stationary states and oscillation amplitudes for transitions between such states. (I am intentionally avoiding the phrase "transition probabilities.") Heisenberg's formalism has developed well and is proving its worth. But it addresses only one side of quantum theory, since the nature of the "transitions" is just as important. Opinions as to the proper approach to this issue are divided. Many feel the problem of transitions is not covered by quantum mechanics in its present form, and that new conceptual formations will be needed. However, it is my belief that the logical structure of quantum mechanics is unified and complete, and thus must include the transition problem. I believe that I have now succeeded in demonstrating this.

Bohr has already drawn attention to the fact that all the fundamental difficulties of quantum ideas which we encounter in the emission and absorption of light by atoms also arise in the interaction of atoms at a short distance, i.e., in collision processes. With these, as opposed to the still-opaque topic of waves and fields, you are dealing exclusively with systems of material particles that are subject to the formalisms of quantum mechanics. I have therefore tackled the problem of investigating more closely the interaction of a free particle ( $\alpha$-ray or electron) and any atom to determine whether a description of the collision process is possible within the framework of current theory.

1 This communication was intended for publication in Naturwissenschaften [The Science of Nature], but could not be included due to lack of space. I hope its publication does not seem superfluous at this point. 
Of the various forms of quantum theory, Schrödinger's has proven the most suitable for this purpose. For this very reason I now regard it as the deepest version of the quantum laws. My train of thought was as follows:

If one wants to quantum mechanically calculate the interaction of two systems, one cannot, as in classical mechanics, single out a state of one system and determine how it is influenced by one state of the other system. Instead, all states of both systems are coupled in a tangled way. That is true also with an aperiodic process, such as a collision, where a particle, say an electron, comes out of the infinite and disappears again in the infinite. But here the idea arises that both before and after the collision, if the electron is far enough away and the coupling is small, it must be possible to define a certain state of the atom and a certain, linear, uniform movement of the electron. The goal is to grasp this asymptotic behavior of the coupled particles mathematically. I did not succeed using my matrix version of quantum mechanics, but I did by using Schrödinger's formulation.

According to Schrödinger, the atom in the $n^{\text {th }}$ quantum state is an oscillation process of a state variable in the whole space with a constant frequency $n \frac{1}{h} W_{n}^{0}$. An electron moving in a straight line is specifically such an oscillation process that corresponds to a plane wave. When both interact, an intricate oscillation arises. But one sees at once that one can fix this at infinity through its asymptotic behavior. You have nothing but a "diffraction problem" in which an incident plane wave is diffracted or scattered at the atom. In place of the boundary conditions used in optics to describe screens we have the potential energy of the interaction between an atom and an electron.

Thus the task is this: We must solve Schrödinger's wave equation for the atom-electron combination under the boundary condition that the solution in a certain direction of the electronic space passes asymptotically into a plane wave of precisely this direction of propagation (the arriving electron). Of the solutions that meet this characterization, we are again mainly interested in the behavior of the "scattered" wave at infinity, since this describes the behavior of the system after the impact. We'll elaborate a bit more on that. Let $\psi_{1}^{0}\left(q_{k}\right), \psi_{2}^{0}\left(q_{k}\right), \ldots$ be the eigenfunctions of the undisturbed atom, for which we assume there is only one discrete sequence. The eigenfunctions $\sin \frac{2 \pi}{\lambda}(\alpha x+\beta y+\gamma z+\delta)$ correspond to the undisturbed (straight) moving 
electron, which form a continuous manifold of plane waves whose wavelength (according to de Broglie) corresponds to the energy $\tau$ of the translational movement, which through $\tau=\frac{h^{2}}{2 \mu \lambda^{2}}$ is linked to $\tau$. The eigenfunction of the undisturbed state in which the electron comes from the $+z$-direction is thus

$$
\psi_{n \tau}^{0}\left(q_{k}, z\right)=\psi_{n}^{0}\left(q_{k}\right) \sin \frac{2 \pi}{\lambda} z
$$

Now let $V\left(x, y, z ; q_{k}\right)$ be the potential energy of the interaction between atom and electron. With the help of simple perturbation calculations one can then show that there is a uniquely determined solution of Schrödinger's differential equation, taking into account the interaction $\mathrm{V}$, which merges asymptotically into the above function for $z \rightarrow+\infty$.

It now depends on how this solution function behaves "after the collision".

The calculation now results: the scattered wave generated by the disturbance has the expression asymptotically at infinity ${ }^{2}$

$$
\psi_{n \tau}\left(x, y, z ; q_{k}\right)=\sum_{m} \int_{\alpha} \int_{x+\beta y+\gamma z>0} d \omega \Phi_{\tau}(\alpha, \beta, \gamma) \sin \underset{\tau}{k n m}(\alpha x+\beta y+\gamma z+\delta) \psi_{m}^{0}\left(q_{k}\right)
$$

That means: the disturbance can be understood in infinity as a superposition of solutions of the undisturbed process. If one calculates the energy belonging to the wavelength $\lambda_{\tau}$ according to the de Broglie's formula given above, one finds

$$
W \underset{\tau}{ }=h v_{n m}^{0}+\tau
$$

where $v_{n m}^{0}$ are the frequencies of the undisturbed atom.

If one wants to reinterpret this result in a corpuscular way, only one interpretation is possible: $\Phi_{\tau} \underset{\tau}{ }(\alpha, \beta, \gamma)$ determines the probability $\left.{ }^{1}\right)$ that the electron coming from the $z$-direction in the one determined by $\alpha, \beta, \gamma$ direction (and with a phase change $\delta$ ) is thrown, whereby its energy $\tau$ has increased by a quantum $h v_{n m}^{0}$ at the expense of atomic energy (collision of

2 Note when correcting: Closer consideration shows that the probability is proportional to the square of the size $\Phi_{\tau}$. 
the first kind for $W_{n}^{0}<W_{m}^{0}, h v_{n m}^{0}<0$; collision of the second kind for $\left.W_{n}^{0}>W_{m}^{0}, h v_{m}^{0}\right)$.

Schrödinger's quantum mechanics therefore gives a very specific answer to the question of the effect of a collision; but it is not a causal relationship. You don't get an answer to the question, "What is the state after the collision", but only to the question, "How probable is a given effect of the collision" (whereby the quantum mechanical energy law must of course be preserved).

Here the whole problem of determinism arises. From the view of our quantum mechanics theory there is no quantity that causally determines the effect of a collision. From the view of experiment we have seen no evidence of internal atomic properties that determine a given collision outcome. Should we hope later to discover properties, such as phases of the internal atomic movements, that determine the individual cases? Or should we instead believe that this remarkable correspondence of theory and experience in our inability to specify conditions for the causal process is a pre-established harmony reflecting the non-existence of such conditions? My tendency is to abandon the atomic world as determined. But that is a philosophical question for which physical arguments alone are not decisive.

In any event, there is in practice indeterminism for both theoretical and experimental and physicists. The "exploitation function" $\Phi$, much studied by experimenters, can now be strictly understood theoretically. It is found in the potential energy of the interaction $V\left(x, y, z ; q_{k}\right)$, though the computational processes required are too involved to communicate here. I just want to explain the meaning of the function $\underset{\tau}{\Phi n}$ in a few words. If $\mathrm{z}$. B. the atom before the collision in the normal state $n=1$, it follows from

$$
\tau+h v_{1 m}^{0}=\tau-h v_{m 1}^{0}=W_{\tau} \cos _{\tau}>0
$$

that for an electron with less energy than the smallest excitation level of the atom it must also be $m=1$, i.e. $W_{\tau}^{11}=\tau$; so there is an "elastic reflection" of the electron with the yield function $\Phi_{\tau}$. If $\tau$ exceeds the first excitation level, there is, in addition to the reflection, excitation with the yield $\Phi_{12}$, etc. If the atom hit is in the excited state $n=2$ and $\tau<h v_{21}^{0}$, there is reflection with the yield $\underset{\tau}{\Phi_{22}}$ and two kinds of collisions with the yield $\underset{\tau}{\Phi_{21}}$. If $\tau>h v_{21}^{0}$, further excitation occurs, etc. 
The formulas completely reflect the qualitative behavior in the event of impacts. The quantitative exhaustion of the formulas for special cases must be reserved for a detailed investigation.

It does not seem to me to be ruled out that the close connection between mechanics and statistics, as it appears here, will require a revision of the basic thermodynamic-statistical concepts.

I also believe that the problem of the irradiation and emission of light must be treated in a completely analogous way as a "boundary value problem" of the wave equation and will lead to a rational theory of attenuation and line width in accordance with the light quantum concept.

A detailed presentation will appear soon in this magazine. 